\title{
Editorial: Sensing and Data Analytics
}

\author{
Benny Raphael ${ }^{1 *}$, Albert Thomas ${ }^{2}$ and Joseph Louis ${ }^{3}$ \\ ${ }^{1}$ Civil Engineering Department, Indian Institute of Technology Madras, Chennai, India, ${ }^{2}$ Department of Civil Engineering, \\ Indian Institute of Technology Bombay, Mumbai, India, ${ }^{3}$ School of Civil and Construction Engineering, Oregon State \\ University, Corvallis, OR, United States
}

Keywords: sensors, data analytics, machine learning, computer vision, monitoring

\section{Editorial on the Research Topic}

\section{Sensing and Data Analytics}

Traditionally, structures have been monitored for ensuring safety and taking appropriate repair or retrofit actions. Strain and accelerometer readings are conventionally used to compare actual structural behavior with the theoretical behavior that is assumed in the design. In many cases, it has been shown that the assumptions made in the design are very conservative and the structure has considerable reserve capacity. Since many developed countries face the problem of aging infrastructure and diminishing funds for new construction, there is increased interest in determining the actual state of the structure, in order to use them in service as far as possible. However, this requires more sophisticated analysis of sensor data; simple comparison of sensor values with permissible limits is not enough. System identification and machine learning techniques are being increasingly used for structural monitoring. Model free data-driven methods are attractive because of the complexities involved in developing accurate models based on physical principles and the difficulties involved in estimating the uncertainties. In particular, there is considerable interest in the application of deep learning techniques to monitoring tasks. In spite of the spectacular success of deep learning in many domains, practical engineering applications are fraught with challenges such as excessive training time required, need for large and clean data sets, and the large number of hyper-parameters to be tuned for optimal performance. From this perspective, new practical applications provide valuable knowledge that helps in the adoption of these sophisticated computational methods to engineering. Another recent development is the use of sensors for applications outside traditional structural monitoring. For example, vibration data is being used to detect occupancy and to infer activities on construction sites. These applications pose new challenges in data interpretation. This special issue on sensing and data analytics aims to cover recent work in this area. This issue contains four research articles focusing on monitoring tasks, two on the application of computer vision and one data report.

The paper by Raphael and Harichandran discusses the challenges in data interpretation due to the mismatch between the actual behavior of the bridge and that assumed in the design. Qualitative visual analysis is important before making use of sophisticated models for data interpretation. It is illustrated how parallel axis plots reveal useful trends in data that help to narrow down on modeling possibilities. Bertola et al. present a framework to include the cost of monitoring into decision making related to sensor placement. The value of information is quantified and is used to justify the cost of measurement. They use the case study of a real bridge to illustrate the approach.

Accurately estimating the loading conditions is hard when controlled load tests are not performed and monitoring is performed while the structure is in service. Wang et al. present computer-vision based methods for use in research on human induced vibrations on pedestrian bridges. The authors conclude that while the precision of computer vision methods is still not high, the approach is promising, and better techniques are continuously being developed due to the intense research happening in this field. 
The article by Riggio et al. is a data report meant to address the lack of open-access monitoring data on moisture response of mass-timber components. In addition to monitoring loading conditions, they present a unique set of hygrothermal data of mass timber elements that had been exposed to changing environmental conditions for almost a year of construction. The data were collected using moisture sensors. Among other benefits provided, the collected data can facilitate the study of hygrothermal performance and support the development of moisture management practices for mass timber products such as cross-laminated timber structural elements.

Overall, the construction industry is witnessing an increased attention toward application of various sensing techniques and machine learning approaches. Due to the unique nature of construction projects where manual intervention/monitoring becomes challenging, innovative sensing approaches become very useful for effective progress monitoring as well as ensuring safety and quality in construction. A study by Delhi et al. in this Research Topic portrays how sensing based interventions can help to better the safety practices in construction projects. Through this study, a novel methodology to monitor the compliance of personal protective equipment (PPEs) through a convolutional neural networks (CNN) based deep learning network is proposed. Similarly, another effort by Nath and Behzadan has developed a CNN based framework to detect various equipment and personnel employed in the construction sites. Rashid and Louis apply machine learning methods to automatically identify the activities performed by articulated construction equipment by analyzing motion data collected from inertial measurement units (IMUs) attached to the various articulated members. These methods aid in sensing the site better with very less manual intervention, and track work progress and measure work productivity. These studies demonstrate the huge potential of machine learning approaches combined with sensing techniques to visualize and interpret data that can eventually augment human capacities in doing construction related tasks.

\section{AUTHOR CONTRIBUTIONS}

All authors listed have made a substantial, direct and intellectual contribution to the work, and approved it for publication.

Conflict of Interest: The authors declare that the research was conducted in the absence of any commercial or financial relationships that could be construed as a potential conflict of interest.

Copyright (c) 2020 Raphael, Thomas and Louis. This is an open-access article distributed under the terms of the Creative Commons Attribution License (CC BY). The use, distribution or reproduction in other forums is permitted, provided the original author(s) and the copyright owner(s) are credited and that the original publication in this journal is cited, in accordance with accepted academic practice. No use, distribution or reproduction is permitted which does not comply with these terms. 